\title{
A rodent model for long-term vagus nerve stimulation experiments
}

\author{
Farid Yaghouby ${ }^{1}$, Benjamin Shafer ${ }^{1}$ \& Srikanth Vasudevan*,1 \\ ${ }^{1}$ US Food \& Drug Administration, Center for Devices \& Radiological Health (CDRH), Office of Science \& Engineering Laboratory \\ (OSEL), Division of Biomedical Physics (DBP), Silver Spring, MD 20993, USA \\ *Author for correspondence: Srikanth.Vasudevan@fda.hhs.gov
}

\begin{abstract}
Aim: Investigations into the benefits of vagus nerve stimulation (VNS) using rodents have led to promising findings for treating clinical disorders. However, the majority of research has been limited to acute timelines. We developed a rodent model for longitudinal assessment of VNS and validated it with a longterm experiment incorporating continuous physiological monitoring. While the primary aim was not to investigate the effects of VNS on the cardiovascular system, we analyzed cardiovascular parameters to demonstrate the model's capabilities in a long-term stimulation-and-recording setup. Materials \& methods: Rats were implanted with a cuff electrode around the cervical vagus nerve and electrocardiogram monitoring devices were implanted in the peritoneal cavity. We also designed a connector mount for seamless access to the cuff electrode for VNS in awake-behaving rats. Results \& conclusion: Results signified easy-to-interface VNS system, electrode robustness and discernible physiological signals in a long-term setup. Analysis of the cardiovascular parameters revealed some transient effects during VNS. Our proposed model enables long-term VNS experiments along with physiological monitoring in unanesthetized rats.
\end{abstract}

First draft submitted: 7 June 2019; Accepted for publication: 13 August 2019; Published online: 16 September 2019

Keywords: bioelectronic medicine $\bullet$ heart rate variability $\bullet$ neuromodulation $\bullet$ physiological monitoring $\bullet$ vagus nerve stimulation

Bioelectronic medicine is an emerging therapeutic approach that employs neuromodulation of peripheral nerves for the treatment of diseases and conditions. The vagus nerve, which is the longest cranial nerve and the primary nerve of the parasympathetic nervous system, has been widely investigated for neuromodulation applications [1-4]. Since the vagus nerve contains both afferent and efferent fibers innervating several organs in thorax and abdomen, electrical stimulation of vagus nerve (known as vagus nerve stimulation or VNS) can modulate function of multiple organ systems [2]. VNS is clinically approved for the treatment of epilepsy, depression and episodic cluster headache (noninvasive VNS) [1,3]. There are numerous applications of VNS currently being studied, such as for the treatment of rheumatoid arthritis [4], tinnitus [5], Crohn's disease [6], stroke [7] and psychiatric disorders [8]. Prior investigations into the mechanisms of VNS have shown the release of neurotransmitters such as acetylcholine and norepinephrine [9] that improves neuroplasticity in sensory and motor cortex when paired with tasks [10]. Applications of VNS-based targeted neuroplasticity training have been studied in rodent models of tinnitus [11], stroke [12] and post-traumatic stress disorder [13] with the potential for clinical translation [14]. Since VNS can have an impact on multiple organ systems, long-term assessment of VNS using animal models provides a deeper understanding of the mechanisms of action and associated off-target effects that will ultimately improve therapeutic outcomes [15-21].

Rodent models have been extensively used to study the mechanisms of action and therapeutic benefits of VNS. For example, the regulatory role of the spleen on immunomodulation through the vagus nerve was discovered using rodent models, which laid the foundation for clinical studies for the treatment of rheumatoid arthritis $[4,16,17]$. Also, anticonvulsive effects of VNS were first characterized using electroencephalography (EEG) experiments from rodent models of epilepsy, that later resulted in implantable devices to control seizures [15,18]. While rodent models have been informative, majority of VNS experiments have been limited to acute timelines [19-27]. Factors such as small animal size, early device failure, device migration and difficult access to electrode connectors can impact 
the experiment duration in rodents [20-32]. While there are prior reports of long-term VNS studies using rodent models [21-35], there still exists an opportunity for improvement.

In this work, we developed a rat model for long-term application of VNS and validated it with continuous physiological monitoring experiment to explore the corresponding effects on the cardiovascular system. We first developed a custom cuff electrode and a plug-and-play connector interface for ease of access. We then refined our surgical protocol for accommodating an implantable physiological monitoring device in the peritoneal cavity, along with a cuff electrode around the left vagus nerve. After animals recovered from surgeries, we applied biweekly VNS in awake-behaving animals without the use of anesthesia and recorded the corresponding electrocardiogram (ECG) from the physiological monitoring devices. Throughout the experimental period, we monitored electrode integrity using impedance measurements. Our results show an easy-to-interface electrical stimulation system, electrode robustness and discernible physiological signals using awake-behaving rats for up to 16 weeks. The proposed animal model facilitates long-term studies to assess the benefits and side-effects of VNS for emerging bioelectronic applications.

\section{Methods}

\section{Animal protocol \& experimental design}

This study was approved by the Institutional Animal Care and Use Committee (IACUC) at the US FDA, White Oak campus. Female Lewis rats $(\mathrm{n}=4,190-205 \mathrm{~g})$ were purchased from Charles River Laboratories International, Inc. Animals were allowed to acclimatize for 1 week with 12-h light and dark cycles upon receipt at the animal facility. As shown in Figure 1A, each rat underwent two successive surgeries separated by 3 weeks to implant: wireless physiological monitoring device (EMKA Technologies, Inc.; Paris, France) for continuous recording of ECG signals (Figure 1B \& C); and custom nerve cuff electrode (Microprobes for Life Sciences; MD, USA) and the transcutaneous connector mount for VNS (Figure 1D \& E). Two weeks following the second surgery, VNS experiment commenced and lasted for 10 weeks based on a defined protocol. Along with VNS, continuous ECG signals (from W1 onward) and intermittent electrode impedance measures (from W4 onward) were recorded from all animals. After 16 weeks, rats were sacrificed, and cuff electrode implant site was exposed to confirm stability and device integrity (Figure 1F).

\section{Electrode design}

The electrode design used in this study consists of two components, a custom nerve cuff and transcutaneous connector mount.

\section{Cuffelectrode design}

A custom bipolar nerve cuff electrode with $1 \mathrm{~mm}$ internal diameter, $12 \mathrm{~cm}$ long lead wire was purchased from Microprobes for Life Sciences. Both electrode contacts were made with $125 \mu \mathrm{m}$ stainless steel wire, with a contact spacing of $1 \mathrm{~mm}$ centered inside a approximately $2 \mathrm{~mm}$ long cuff (Figure 2C). To aid with closing the cuff after implantation, 8-0 silk sutures were attached by the manufacturer as shown in the Figure 2C. The two leads were connected to a 4-pin female Mill-Max connector (Mouser Electronics; TX, USA).

\section{Connector mount design}

A two-part connector mount was designed as described elsewhere [22]. The connector mount was designed using SolidWorks (Dassault Systèmes SolidWorks Corp.; MA, USA). The parts were then 3D printed (www.shapeways. com), with the bottom part made of Nylon and the top made with bronze infused stainless-steel (Figure 2A). The top part was designed to incorporate strong magnets $(3 \times 1 \mathrm{~mm}$ disc, Neodymium Magnet, Apex Magnets [WV, USA]). The two parts were attached using epoxy (Loctite Hysol epoxy). A $2 \times 2 \mathrm{~cm}$ Mersilene mesh was attached to the bottom of the connector mount using epoxy to promote tissue ingrowth after implantation (Figure 2C). The connector from the cuff electrode was then secured inside the connector mount as shown in Figure $2 \mathrm{C}$ using epoxy. Finally, a layer of Kwik-cast (World Precision Instruments, Inc. [FL, USA]) was applied to the bottom of the connector mount to provide soft tissue contacting surface. To protect lead wires from mechanical stress after implantation, a $2.5 \mathrm{~cm}$ long silicone tube (United States Plastic Corp. [OH, USA]) was added around the wires at the connector interface (Figure 2C). An external connector holder was designed, and 3D printed with Nylon to interface with the transcutaneous connector mount (Figure 2B). The magnets on this part were aligned with the connector mount for attachment. A 4-pin Mill-Max male connector (Mouser Electronics) was centered and 


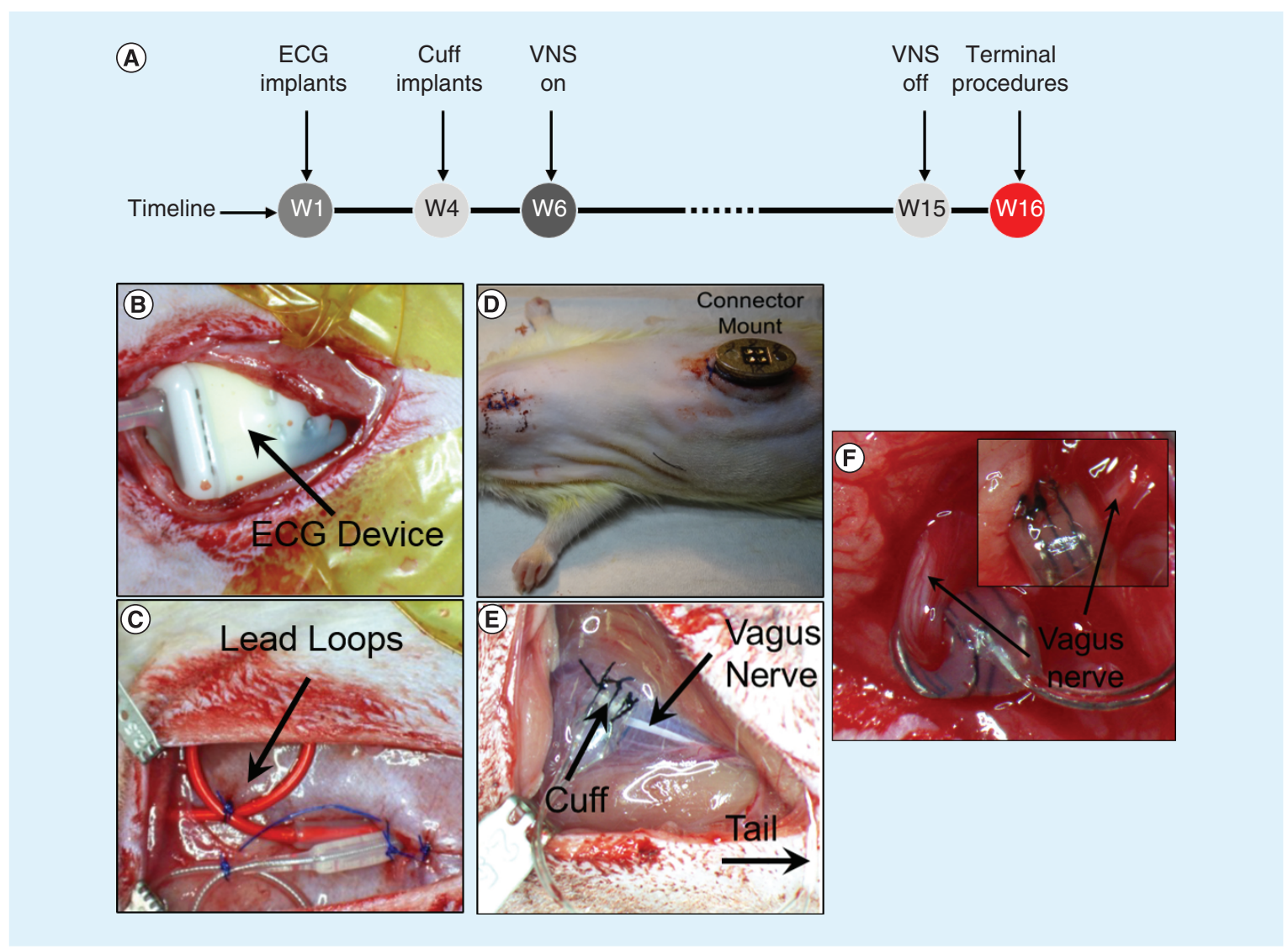

Figure 1. Experimental design and surgical implantations. (A) Timeline for the experiment. Total experiment length is 16 weeks (W1-16) including 10 weeks of VNS. (B) First surgery at W1: wireless ECG device is inserted inside the peritoneal cavity. (C) Electrode leads from ECG device were looped to minimize any potential damage due to mechanical tension. (D) Second surgery at W4: a connector mount is surgically affixed to the lumbar fascia. (E) Cuff electrode is implanted around the left cervical vagus nerve. (F) Terminal procedure at W16 showing nerve inside the cuff electrode.

ECG: Electrocardiogram; VNS: Vagus nerve stimulation.

Figure 2. Vagus nerve stimulation cuff electrode and connector mount design. (A) Fully assembled connector mount with a 4-pin female connector shown next to it. Connector mount consists of two parts: a bottom part (Nylon) with suture holes and a top part (stainless steel) which contains magnets. (B) External connector holder is made of plastic with two magnets and male connector for ease of interfacing with the connector mount. (C) Fully assembled implant with cuff electrode attached to the connector mount. Higher magnification image showing the 2-contact cuff electrode.

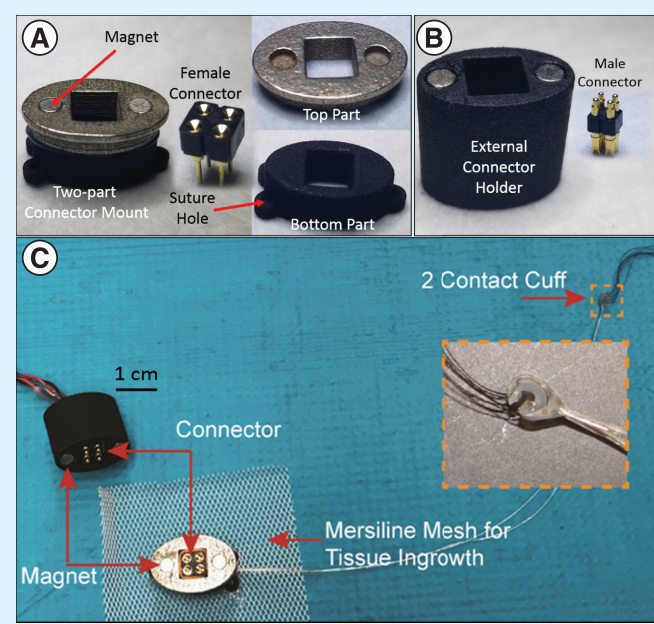


attached using epoxy. The pins from the male connector were extended out by approximately $1 \mathrm{~mm}$ for achieving optimum contact with the female connector (Figure 2A \& B). Finally, the connections were thoroughly tested for electrical continuity.

\section{Surgical procedures}

To anesthetize rats, a cocktail of ketamine $\left(75 \mathrm{mg} \mathrm{kg}^{-1}\right)$ and dexmedetomidine $\left(0.25 \mathrm{mg} \mathrm{kg}^{-1}\right)$ was administered (intraperitoneal injection). After confirming the lack of firm hind toe pinch response, analgesic agent (Meloxicam; 1-2 $\mathrm{mg} \mathrm{kg}^{-1}$ ) was injected subcutaneously. Intramuscular (im.) injection of antibiotic (Cefazolin $15 \mathrm{mg} \mathrm{kg}^{-1}$ ) was administered prior to the first incision, and every $1.5-2 \mathrm{~h}$ thereafter until recovery from anesthesia, if needed. Surgical site was shaved and thoroughly disinfected with betadine and alcohol. To prevent dehydration, $3 \mathrm{ml}$ of warm sterile saline (Medline Addipak; IL, USA) was administered subcutaneously. Ocular ointment was applied to prevent post-surgical discomfort. To prevent hypothermia during the procedure, animals were placed on a heating pad (circulating water) and the body temperature was closely monitored and maintained using a rectal temperature probe (Darvall Vet; Gladesville, NSW, Australia). In addition to temperature, other vital signs including heart rate and arterial oxygen saturation $\left(\mathrm{SpO}_{2}\right)$ were continuously monitored using a rat pulse oximeter and heart rate monitor (Kent Scientific Corp.; CT, USA). An additional dose of antibiotic (Cefazolin $15 \mathrm{mg} \mathrm{kg}^{-1}$ ) was also injected into each incision site, just prior to wound closure. Finally, antibiotic topical ointment was applied over the incisions to prevent infections. Animals were then administered Atipamezole $\left(0.5 \mathrm{mg} \mathrm{kg}^{-1}\right.$, ip.) for anesthesia reversal and returned to their home cages and monitored for normal locomotion until fully recovered. For further infection control, Trimethoprim-sulfamethoxazole (sulfatrim; $3 \mathrm{ml}$ of $144 \mathrm{mg}$ sulfatrim in 9 oz water) was administered for 5 days post-surgery. To manage pain, analgesic (Meloxicam; $1-2 \mathrm{mg} \mathrm{kg}^{-1}$ ) injection was repeated for days 2 and 3 post-surgery. Sutures were removed 10-14 days after surgery to ensure complete healing of the incision sites. At the end of the experiment (week 16) rats were first anesthetized and then euthanized for device harvest.

Physiological monitoring device surgery

A 5-6 cm lateral skin incision in the abdomen was made followed by blunt dissection to expose the peritoneal cavity. The wireless physiological monitoring device (EMKA technologies Inc.; Paris, France) was inserted into the peritoneal cavity and sutured to the abdominal muscles (4-0 polypropylene suture) as shown in Figure 1B. The two leads from the implant were looped first and then inserted into chest muscle using an $18 \mathrm{G}$ needle serving as a cannula, one lead on each side and secured using sutures (4-0 polypropylene suture). The procedure was completed by closing muscles and all skin incisions using polypropylene sutures and GLUture (World Precision Instruments; FL, USA). The entire surgical procedure lasted for approximately $1 \mathrm{~h}$. Animals were recovered after the surgical procedure as described previously.

\section{Cuff electrode implantation}

Three weeks after implanting the physiological monitoring devices, animals were implanted with a cuff electrode around the left cervical vagus nerve. First, an incision was made over the lumbar area to secure the connector mount as described elsewhere $[22,23]$ followed by an incision over the neck area to tunnel the lead. The vagus nerve was then exposed and was carefully dissected and freed from surrounding connective tissue within the carotid sheath [11]. Saline was added to all incisions to maintain tissue hydration. The connector mount was sutured to lumbar fascia and the cuff electrode was tunnel to the site of implantation (Figure $1 \mathrm{D} \& \mathrm{E}$ ). The nerve was carefully placed into the cuff electrode using fine forceps and fibrin sealant was applied (Ethicon; NJ, USA) to stabilize the electrode nerve construct. After fibrin sealant fully cross-linked, the muscle implant site was closed with suture followed by skin closure with a combination of suture and GLUture. To complete the surgical procedure, the lead was looped on the back of the neck and secured with a suture and all incisions were closed with a combination of suture and GLUture (Figure 1D). The entire surgical procedure took approximately $2 \mathrm{~h}$.

\section{Data collection \& stimulation ECG recording}

Continuous ECG recording commenced on each rat following implantation of the physiological monitoring implant. ECG signals were continuously acquired using a wireless telemetry system (EMKA Technologies, Inc.) at $500 \mathrm{~Hz}$ sampling frequency. Data were stored in a PC and later converted to the European data format for further analysis. 


\section{VNS protocol}

Cuff electrode integrity was confirmed with impedance measurement starting at week 4 (W4). Two weeks after recovery from the second surgery, VNS protocol was started on W6 and continued for 10 weeks. An external electrical stimulator was used for applying VNS in awake-behaving rats (A-M Systems; WA, USA). VNS was delivered twice a week (Tuesday and Friday) based on fixed parameters: constant-current, biphasic and charge balanced square wave pulses ( $1 \mathrm{~mA} /$ phase, $100 \mu \mathrm{s} /$ phase $)$ at $30 \mathrm{~Hz}$ applied for $30 \mathrm{~s}$. Before interfacing the external connector to the connector mount, both connectors (implanted and external) were cleaned with alcohol pads to ensure connectivity. After attaching the connectors, the stimulator was turned on to apply VNS, while animals moved freely inside the cage.

\section{Impedance measurement}

Electrode impedance was recorded at $1 \mathrm{kHz}$ (Gamry Instruments, Inc.; PA, USA) from awake-behaving rats to confirm integrity of the leads. Prior to implantation, each cuff electrode was submerged in phosphate-buffered saline to record pre-implant impedance between the two leads. After implantation, impedance measurements were recorded immediately (W4) and weekly thereafter to confirm electrode integrity. Weekly impedance measurements continued for up to 13 weeks (including 10 weeks of VNS) on each animal.

\section{Device explant}

Durability of the nerve cuff electrode for long-term VNS experiments was also verified by harvesting the device at the end of the experiment. Animals were sacrificed, and implant area was carefully dissected to observe cuff electrode around the vagus nerve (Figure $1 \mathrm{~F}$ ).

\section{Data analysis}

Assessment of electrode integrity

To ensure that vagus nerve cuff electrodes remain intact after several weeks of stimulation in awake-behaving animals, impedance measurements were recorded on a weekly basis throughout the experiment. Magnitude of measured impedance at $1 \mathrm{kHz}$ was selected from the recorded spectrum and analyzed. At the end of the experiment (i.e., W16), animals were sacrificed, and electrodes were inspected to confirm stability around the nerve.

\section{ECG features}

To study the cardiovascular effects of VNS, continuous ECG signals recorded by data acquisition system were first converted to European data format and then pre-processed and analyzed for waveform detection using LabChart Pro (ADInstruments; CO, USA) software. Default parameters for rat ECG analysis were used on LabChart Pro to remove abnormal waveform detections such as ectopic beats (beats with estimated heart rate less than $250 \mathrm{bpm}$ or more than $500 \mathrm{bpm}$ were excluded). Beat-to-beat ECG waveform detections were exported in comma separated vector (CSV) files for ECG morphology and heart rate variability (HRV) analysis using MATLAB (Mathworks, Inc.; MA, USA). To explore possible effects of the second surgery on cardiovascular system, we compared weekly heat rate $(\mathrm{HR})$ trends for every animal before and after implantation of the cuff electrode. As shown in Figure 3A, HR dynamic range and circadian variation in light-dark cycles remained unchanged after cuff electrode implantation, indicating no significant change in behavior and activity resulted from surgeries. Furthermore, comparison of selected HRV parameters before and after the cuff electrode implantation did not reveal any significant differences (Figure 3B). In addition to these evidences from recorded ECG data, our animals were closely monitored following each surgery to confirm complete recovery, normal behavior and overall welfare.

For ECG morphology, two waveform features that are physiologically meaningful and less susceptible to baseline noise and detection errors were averaged in 15-min segments. As seen from Figure 3D, the time between P-wave onset to QRS onset (PR interval) and the time between QRS onset and end of T-wave (QT interval) were selected as ECG morphology features. By convention, QT interval is usually reported as corrected QT (QTc), since QT interval has an inverse relationship to the instantaneous HR. Here, the Bazelett normalization method was used to normalize QT measures to RR interval $\left(Q T c=\frac{Q T}{\sqrt{R R}}\right)$. In addition to ECG morphology parameters, to study the autoregulation of heart rhythmicity by autonomic nervous system, HRV variables [24] were extracted from 15-min segments of RR interval time-series using custom MATLAB codes. Three groups of HRV features were extracted from 15-min segments of RR interval time-series for each recording, namely time-domain, frequency-domain and 
(A)

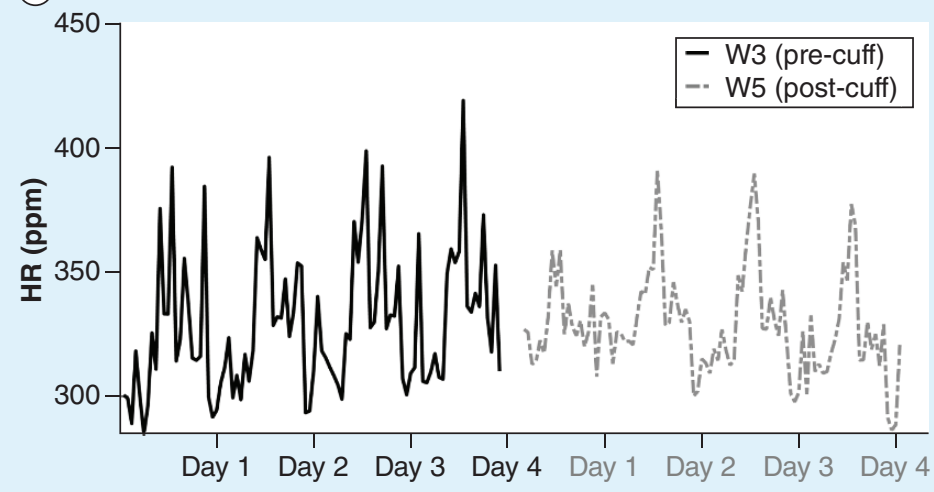

(c)

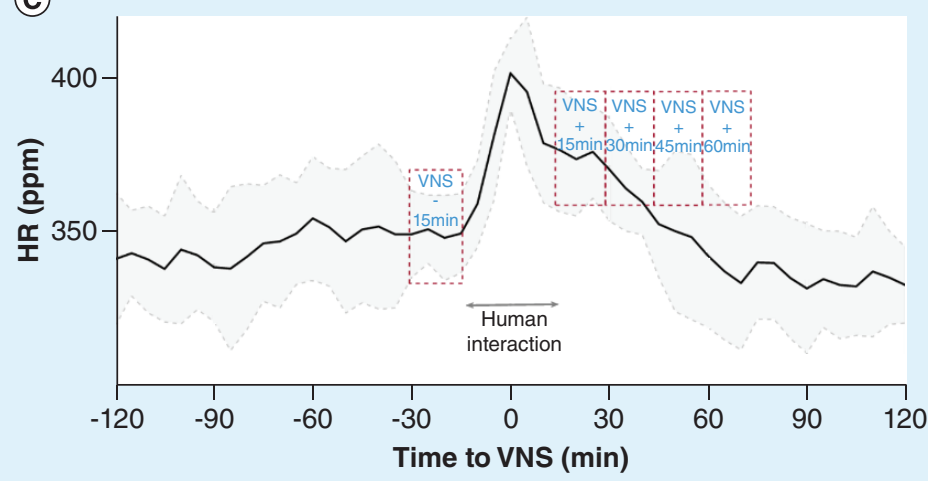

(B)
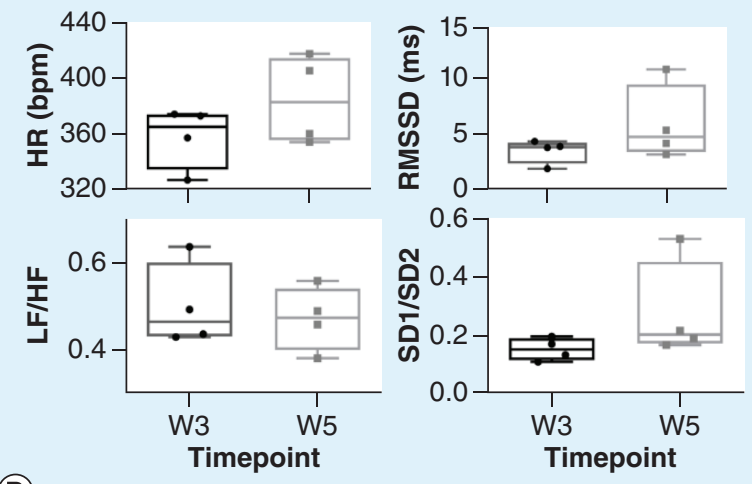

(D)

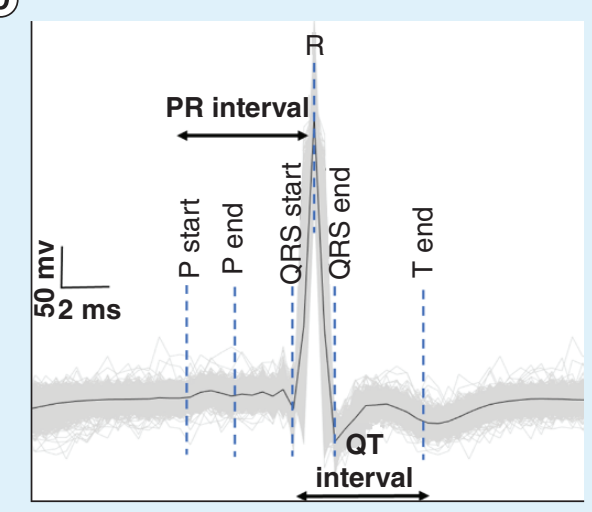

Figure 3. Electrocardiogram analysis. (A) HR trends before and after cuff electrode implantation. HR variations during light and dark cycles are evident and did not change after surgery. (B) Comparison of selected heart rate variability parameters before and after cuff electrode surgery did not show any significant changes. (C) Average heart rate trend for all VNS sessions from four rats (mean $\pm 95 \% \mathrm{Cl}$ ). An early tachycardia is evident due to human interaction during the stimulator connect/disconnect procedures. (D) Average ECG waveform recorded from one animal shows that morphology remains evident even at week 16.

HF: High frequency; HR: Heart rate; LF: Low frequency; RMSSD: Square root of the mean squared differences of successive RR intervals; VNS: Vagus nerve stimulation.

nonlinear dynamics. As time-domain features, average HR, standard deviation of RR intervals (SDNN), square root of the mean squared differences of successive RR intervals (RMSSD) and baseline width of the RR interval histogram (TINN) were calculated. As frequency-domain features, absolute power of the low-frequency (LF: 0.2$0.75 \mathrm{~Hz}$ ) and high frequency (HF: $0.75-2.5 \mathrm{~Hz}$ ) bands as well as the power ratio (LF/HF) were calculated using Lomb-Scargle periodogram estimation of RR interval time series. Nonlinear analysis of HRV-included Poincare plot, detrended fluctuation analysis (DFA) and entropy estimation. To perform Poincare plot analysis for assessment of self-similarity in RR interval time series, each RR interval is first plotted against next RR interval. Then, standard deviation of RR intervals along the line perpendicular to the line of identity in Poincare plot (SD1) and standard deviation of RR intervals along the line of identity in Poincare plot (SD2) were calculated [24]. For DFA analysis, the $\log -\log$ slope in the low-scale $(\alpha 1)$ and high-scale $(\alpha 2)$ regions were estimated to reflect brief and long-term RR fluctuation, respectively. Also, sample entropy ( $\mathrm{SaEn}$ ) was calculated to assess the predictability of fluctuations in successive RR intervals from the analyzed data sample.

\section{Statistical analysis}

Data are presented as mean \pm standard error. Statistical analyses were performed using GraphPad Prism software (V8). One-way analysis of variance (ANOVA) with repeated measures was used to perform statistical comparisons between baseline and post-VNS data points. Values of $\mathrm{p}<0.05$ were statistically significant. If appropriate, post hoc comparisons with Dunnett's test were performed, with p-values corrected for repeated comparisons. 
Figure 4. Electrode impedance monitoring over the course of the experiment (mean \pm standard error of the mean). Impedance magnitude at the $1 \mathrm{Khz}$ shows a relatively stable trend after some increase at the first few weeks $(n=4)$. Impedance data from one animal were removed at the end point (W16) due to relatively large value $(|z|>300 \mathrm{k} \Omega)$.

*Indicates significant difference compared with W4 ( $p<$ 0.05).

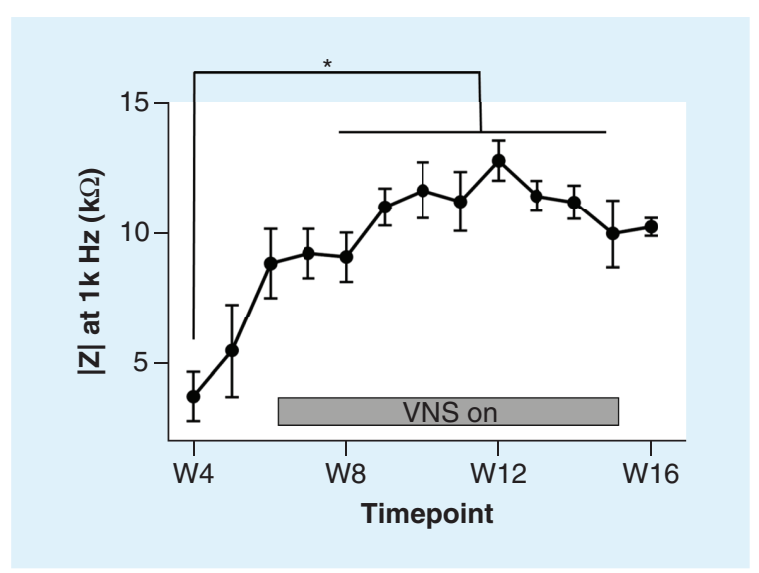

\section{Results}

\section{Cuff electrode integrity}

Weekly measurements of impedance $(1 \mathrm{kHz})$ showed a slight increase for the first 2 weeks and a relatively stable trend $(5 \mathrm{k} \Omega<|\mathrm{z}|<15 \mathrm{k} \Omega$ ) thereafter (Figure 4). One rat with abnormally high impedance measurement ( $>300 \mathrm{k} \Omega$ ) at the end point (W16) was removed from the graph. Overall, impedance measurements confirmed that implanted electrodes remained functional for long-term use, although from W8 onward, the measured impedance was significantly higher (but still within acceptable range) compared with the beginning (i.e., W4). At the end of W16, animals were sacrificed, and electrodes stability around the vagus nerve was confirmed. Figure $1 \mathrm{~F}$ shows a representative cuff electrode around the vagus nerve at the time of explantation (W16). As can be seen, the cuff electrode is encapsulated, with the vagus nerve inside the cuff throughout the experimental period. Impedance measurements and harvest observations confirmed that the custom cuff electrode and our surgical procedures are suitable for long-term VNS experiments.

\section{Cardiovascular effects of VNS}

Cardiovascular effects of VNS were preliminarily assessed by analyzing ECG signals recorded from four rats during 10 weeks of stimulation (W6-15). Due to early battery failure in physiological monitoring devices, ECG signals were only available for 5 and 6 weeks of VNS in the first two rats, respectively. Average HR trend (estimated from 5-min ECG segments) time-locked to the VNS onset shown in Figure 3C demonstrated that all rats experienced tachycardia following VNS with approximately $1 \mathrm{~h}$ recovery time. However, the tendency seems to be started before triggering VNS and on average, HR increased by $10-15 \%$ from time -15 to time 0 (Figure 3C). This early trend is due to the interaction between experimenter and animals during VNS sessions. To minimize the confounding effects of such factors from our analysis, a 30 min window of ECG signals centered at the VNS onset (time 0) was excluded from all ECG morphology and HRV analyses to avoid using data recorded in the presence of the experimenter. In Figure 3C, data proportions for baseline (VNS-15 min) and post-VNS (VNS+15, 30, 45 and $60 \mathrm{~min}$ ) are marked. ECG morphology features along with HRV analyses have been performed to investigate short-term and long-term effects. To study short-term effects, each cardiovascular parameter was compared for baseline and post-VNS segments. For long-term analysis, each variable estimated from the first post-VNS window (VNS +15 min) was first normalized with respect to the baseline (VNS-15 min) and then the trend was presented over the course of the VNS experiment (W6-15).

\section{ECG morphology features}

Two morphology features shown in Figure 3D were selected and computed (from 15-min ECG segments) for comparison. Our preliminary investigation revealed that these two ECG features are more robust to baseline noise compared with others and the quality of ECG remains acceptable to estimate those with relatively high confidence. In Figure 5A and B, short-term trends for PR and QTc were shown for baseline (VNS - $15 \mathrm{~min}$ ) and four timepoints post-VNS, respectively. PR reflects the impulse conduction from atria to ventricles and prolonged PR may indicate first degree heart block. As shown here, PR remained unchanged right after VNS with a slowly increasing trend over time without any significant differences with respect to the baseline (Figure 5A, p > 0.05). Ventricular activity time reflected by QTc slightly increased following VNS and recovered gradually to the baseline level within 

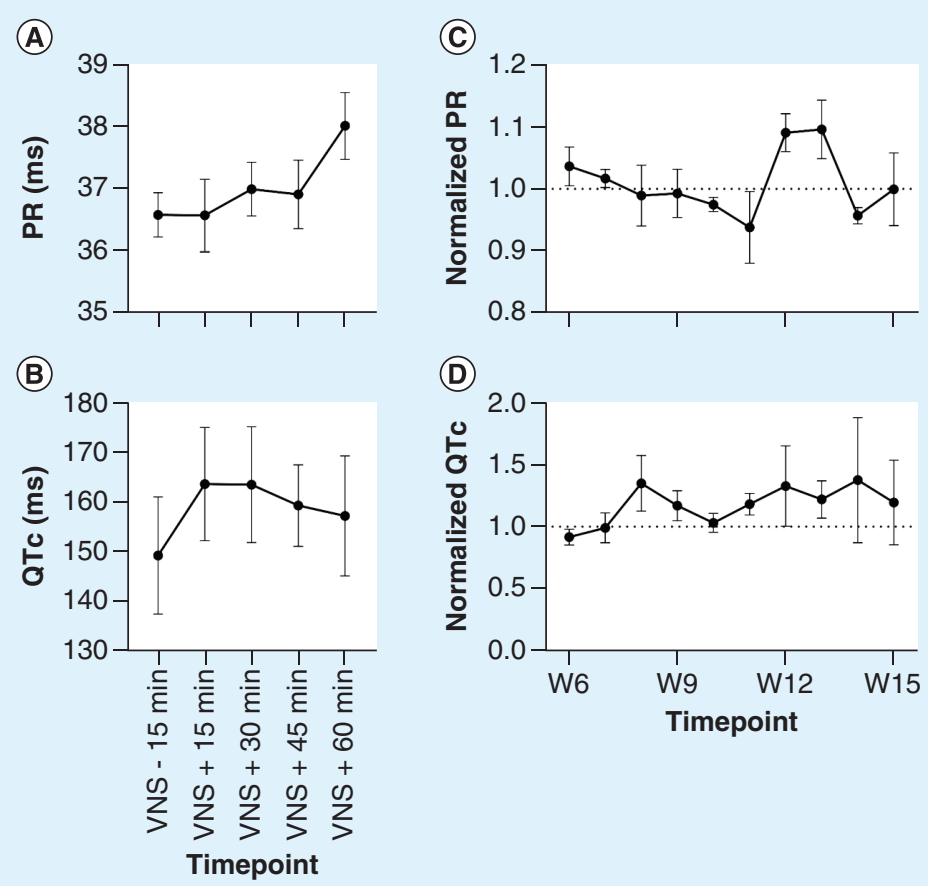

Figure 5. Electrocardiogram morphology feature trends (mean \pm standard error of the mean). (A \& B) Short-term trends for PR and QTc remained mainly unchanged following vagus nerve stimulation with slightly increasing trend over time $(n=4)$. QTc slightly increases by vagus nerve stimulation (not significant) and recovers within 1 h. (C \& D) Long-term trends for PR and QTc are opposite but not significant ( $n=4$ for W6-10, $n=3$ for W11 and $n=2$ for W12-15). QTC: Corrected QRS onset and end of T-wave; PR: Time between P-wave onset to QRS onset.

$1 \mathrm{~h}$ (Figure 5B). However, ANOVA test did not reveal any significant effects of VNS on QTc here ( $\mathrm{p}>0.05)$. Long-term effects of VNS were examined by tracking weekly values of normalized PR and QTc (Figure 5C \& D). Normalized PR gradually decreased and reached to the below baseline level after 5 weeks of VNS. The longitudinal trend for W11-15 is not consistent; because of limited sample size from W11 onward. Due to premature battery failure for ECG wireless device, sample size at different time points of Figure 5C and D is $n=4$ (W6-10), $n=3$ (W11) and $\mathrm{n}=2$ (W12-15). Normalized QTc raised above baseline level after 3 weeks of VNS and then remained mainly unchanged (Figure 5D). Statistical comparison (only applied up to W10 where full sample size was available) did not show any significant long-term effects.

\section{HRV time-domain features}

Figure 6 summarizes the short-term and long-term effects of VNS on HRV time-domain features. On the left side (Figure 6A-D), sustained tachycardia and transient short-term HRV effects were significant $(\mathrm{p}<0.05)$. HR remained high for up to $1 \mathrm{~h}$ post-VNS while RMSSD increased by almost 50\% post-VNS with quick recovery (Figure 6A \& C). SDNN did not change and remained constant except for a slight decrease 30 min postVNS (Figure 6B). TINN also showed a transient and nonsignificant increase by approximately $25 \%$ post-VNS. Statistically significant differences were found for HR and RMSSD when compared with the baseline for multiple time points post-VNS $(\mathrm{p}<0.05)$. The right panel of Figure 6 compares the long-term effects of VNS for normalized variables, over the course of 10 weeks VNS. Post-VNS normalized variables did not show clear trends for SDNN, RMSSD and TINN, although they remained mainly above baseline levels (Figure 6F-H). However, normalized HR consistently increased until W9 and then plateaued thereafter (Figure 6E). Abnormal values for W11-15 are because data from two rats were removed due to the lack of ECG signals after battery failure. Statistical assessment was only performed up to W10 where full sample size $(\mathrm{n}=4)$ was available for long-term trends. Only normalized HR showed significant difference at W8 compared with W6. 

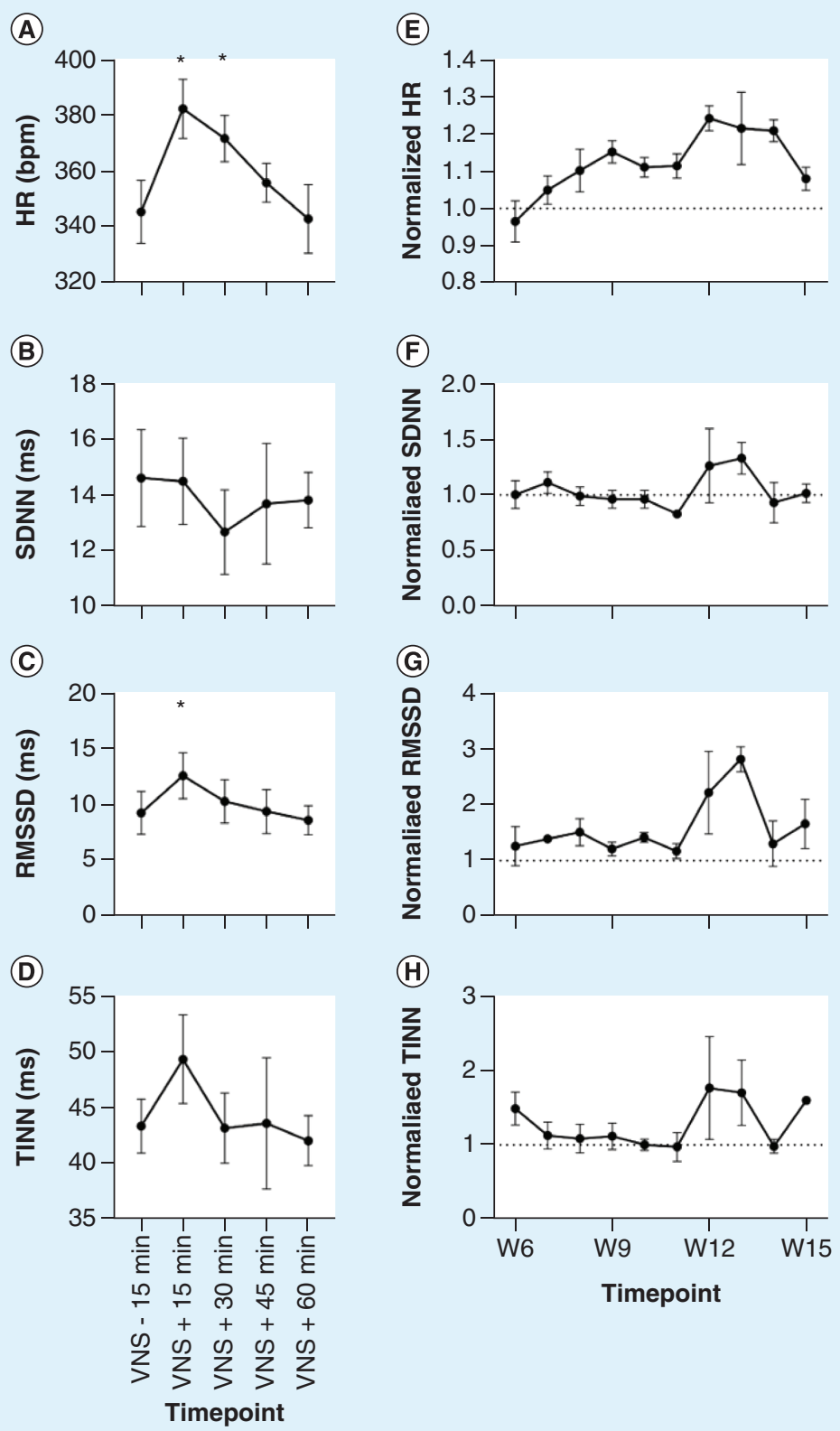

(H)

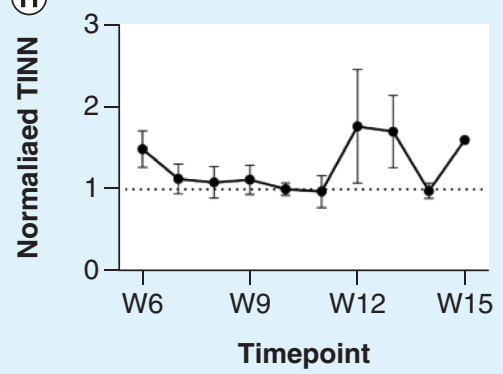

Figure 6. Heart rate variability time-domain features (mean \pm standard error of the mean). (A-D) Short-term trends for HR, SDNN, RMSSD and TINN. Sustained tachycardia and transient short-term variability are evident $(n=4)$. $(E-H)$ Long-term effects of VNS on heart rate variability time-domain features. Post-VNS variables mainly remained unchanged except HR ( $n=4$ for W6-10, $n=3$ for W11 and $n=2$ for W12-15).

*Indicates significant difference with respect to VNS - 15 min at left panel or W6 at right panel $(p<0.05)$.

HR: Heart rate; SDNN: Standard deviation of RR intervals; TINN: Baseline width of the RR interval histogram; VNS:

Vagus nerve stimulation.

\section{HRV frequency-domain features}

Power spectral analysis of RR interval signals was summarized as three features shown in Figure 7. As shown in the left panel of Figure 7, LF and HF powers both increased slightly right after VNS and given the LF/HF trend, the contribution of vagal activity (reflected as HF) seemed to be larger. Although no statistically significant effect was observed, suppression in LF/HF indicating parasympathetic dominance lasted longer and the recovery to baseline level was evident after about $45 \mathrm{~min}$ (Figure 7A-C). As long-term effects, normalized LF and HF decreased at 
(A)

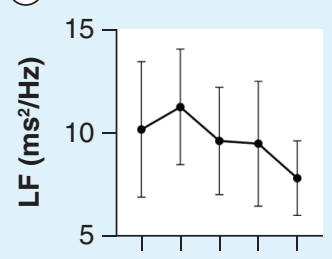

(B)

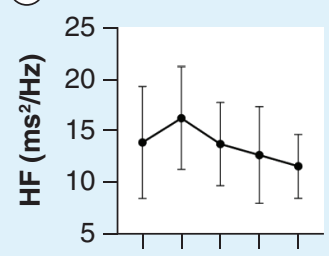

(C)

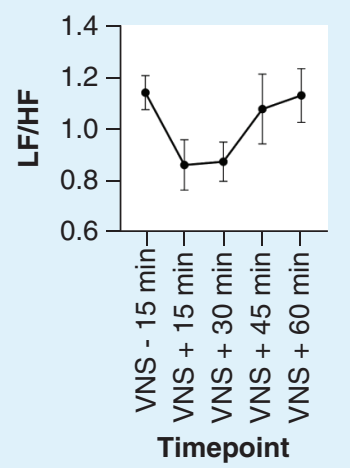

(D)

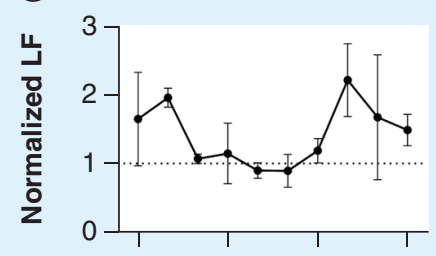

(E)

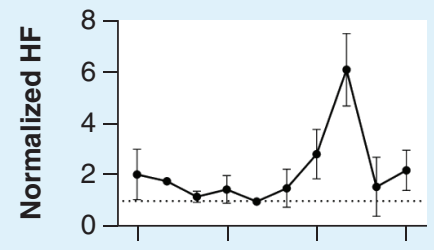

(F)

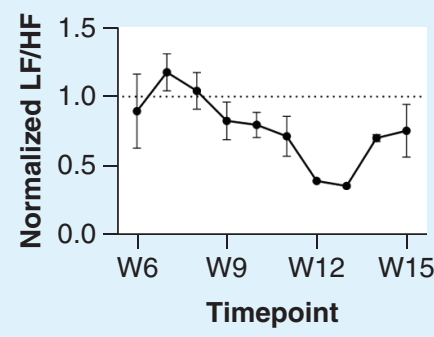

Figure 7. Heart rate variability frequency-domain features (mean \pm standard error of the mean). (A-C) Short-term trends for LF, HF and LF/HF. Parasympathetic modulation is predominant after VNS $(n=4)$. (D-F) Long-term effects of VNS on heart rate variability frequency-domain features. LF/HF slowly decreases over time ( $n=4$ for W6-10, $n=3$ for $\mathrm{W} 11$ and $\mathrm{n}=2$ for W12-15).

HF: High frequency; LF: Low frequency; VNS: Vagus nerve stimulation.

the beginning and then remained relatively unchanged at the baseline level with slight increase toward the end (Figure 7D \& E). The normalized LF/HF increased initially and then decreased and remained well below baseline (not significant) between W6-15. Dynamic range of values for W12\&13 seemed to be different from others possibly because of limited sample size.

\section{$H R V$ nonlinear features}

As shown in the left panel of Figure 8, variability in nonlinear dynamics following VNS was primarily observed in SD1/SD2 and SaEn $(p<0.05)$. Unlike SaEn, SD1/SD2 recovered to the baseline level after approximately $1 \mathrm{~h}$ post-VNS. Correlation between successive RR intervals over different time scales extracted by DFA $(\alpha 1 / \alpha 2)$ showed a marginal surge following by quick recovery after VNS (Figure 8B). ANOVA comparison did not reveal any significant differences for post-VNS $\alpha 1 / \alpha 2$ compared with the baseline level ( $\mathrm{p}>0.05)$. The long-term trend for normalized variables showed a gradual increase in SD1/SD2 and relatively constant trend for $\alpha 1 / \alpha 2$ and SaEn (Figure 8D-F). No significant long-term effect was found for normalized variables.

\section{Discussion}

One of the major translational challenges in development of animal models is the ability to imitate human physiology. However, information from animal models along with clinical findings provide better translational insights on device safety and efficacy [25]. Long-term VNS experiments in animal models, particularly using rodents 
(A)

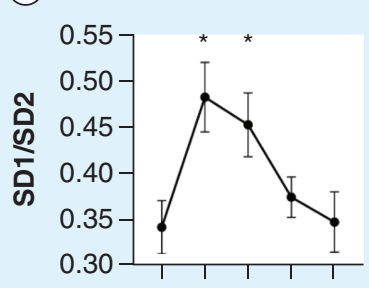

(B)

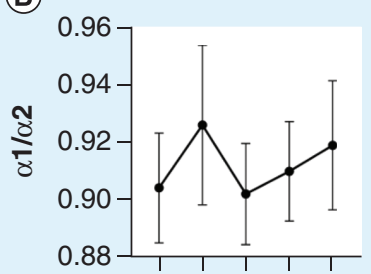

(C)

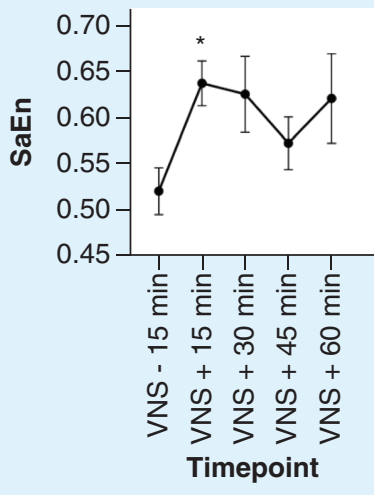

(D)

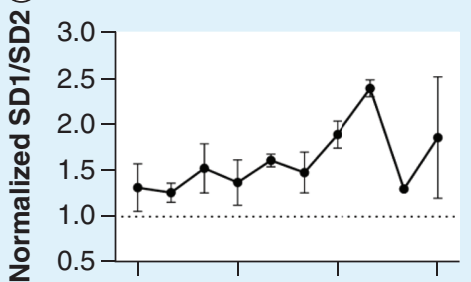

(E)

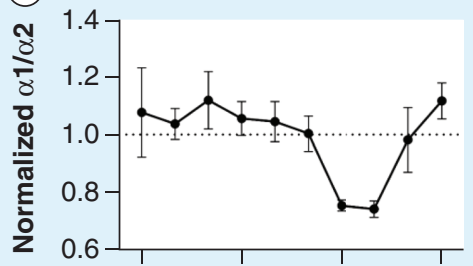

(F)

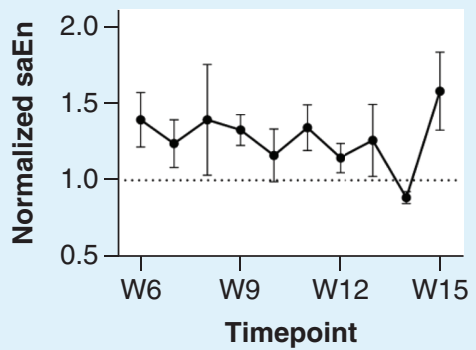

Figure 8. Heart rate variability nonlinear features (mean \pm standard error of the mean). (A-C) Short-term trends for SD1/SD2, $\alpha 1 / \alpha 2$ and SaEn. Short-term/long-term viability increases over time as reflected on SD1/SD2 $(n=4)$. (D-F) Long-term effects of VNS on heart rate variability nonlinear features. Normalized SD1/SD2 slightly increases over time ( $\mathrm{n}=4$ for W6-10, $\mathrm{n}=3$ for $\mathrm{W} 11$ and $\mathrm{n}=2$ for W12-15).

*Indicates significant difference with respect to the baseline $(p<0.05)$.

VNS: Vagus nerve stimulation.

are technically challenging, majorly due to small animal size and early failure or migration of implanted device. To enhance the clinical relevance and extrapolation of experimental data to humans, considerations such as long-term VNS experiments using unanesthetized and freely moving animals [15], clinically relevant parameter space for stimulation $[26,27]$ and application of robust electrodes and implants [28] should be taken into account.

In this study, the feasibility of long-term VNS along with ECG monitoring in rats for up to 4 months (including 10 weeks of stimulation) were assessed. Augmenting VNS cuff electrode implantation with another surgery to place a physiological monitoring device can be challenging, as it can lead to early device failure or animal discomfort. Following proposed surgical procedures, rats tolerated two device implantation surgeries and round-the-clock physiological signals were recorded wirelessly over long periods of time. Failure-related ECG implants during the experiments were independent of our surgical techniques and were associated with early battery failure. Hence, we were able to report data from two animals for less than 10 weeks of VNS (5 and 6 weeks, respectively). ECG signal quality remained suitable for estimating both morphology and HRV features even at 4 months post-implantation; indicating that the surgical procedures developed in this study are conducive for developing ECG based closed-loop VNS studies. No adverse events were observed in this study and the same outcome can be reproduced by repeating the protocol for other applications. The optimal outcomes resulted from refining the anesthetic regimen and surgical techniques that we learned through preliminary investigations. 
Table 1. Summary of selected long-term vagus nerve stimulation experiments in rats.

\begin{tabular}{|c|c|c|c|c|c|c|}
\hline Model & Measurement & Duration & VNS rate & Animal status & Assessment & Ref. \\
\hline Stroke & Behavior & 6 weeks & Seconds/day & Awake & NM & [12] \\
\hline PTSD & Behavior & 11 days & Every other day & Awake & NM & [13] \\
\hline Control & Acute EFP & 6 days & Once on day 4 & $\begin{array}{l}\text { Awake } \\
\text { (VNS)/anesthetized } \\
\text { (EFP) }\end{array}$ & NM & [38] \\
\hline Heart failure & $\mathrm{ECG}+\mathrm{BP}$ & 6 weeks & $10 \mathrm{~s}$ on $/ 50 \mathrm{~s}$ off & Awake & NM & [31] \\
\hline Ovariectomized & Blood analysis & 12 weeks & $3 \mathrm{~h} /$ every other day & Awake & NM & [21] \\
\hline Heart failure & Echo + blood analysis & 8 weeks & NM & Awake & NM & [39] \\
\hline Hypertension & $\mathrm{ECG}+\mathrm{BP}$ & 4 weeks & $1 \mathrm{~h}$ on $/ 1 \mathrm{~h}$ off & Awake & $\begin{array}{l}\text { ECG target } \\
\text { engagement }\end{array}$ & [29] \\
\hline Epilepsy & EEG & 6 days & $2 \mathrm{~h} /$ day & Awake & $\begin{array}{l}\text { Impedance } \\
\text { measurement }\end{array}$ & [33] \\
\hline PAH & $E C G+B P$ & 5 weeks & NM & Awake & NM & [34] \\
\hline
\end{tabular}

BP: Blood pressure; ECG: Electrocardiogram; Echo: Echocardiography; EFP: Evoked field potential; NM: Not mentioned; PAH: Pulmonary arterial hypertension; PD: Parkinson's disease; PTSD: Post-traumatic stress disorder.

The explant procedure showed that our custom design for nerve cuff electrode (e.g., diameter size and lead length) was appropriate for long-term use (Figure $1 \mathrm{~F}$ ). Also, electrode leads were adequately sturdy to tolerate repeated neck motion for several weeks. The chosen location to fix the connector mount provided two major benefits: it was less susceptible to animal inflicted damage and was a convenient location for the experimenter to connect the stimulator in awake-behaving animals (Figure 1D). The magnetic feature of the connector mount provided convenient plug-and-play interface without the need to anesthetize the animals for applying stimulation (Figure 2). In this study, behavioral changes such as coughing, freezing and muscle twitches were also observed in response to stimulation.

The main objective of this study was to develop a rodent model for long-term assessment of VNS. Although cardiovascular effects of VNS have been studied here, neither experimental design nor sample size was determined a priori to examine the physiological effects or interpret the underlying mechanisms. Influence of VNS on HRV resulting from elevated vagal tone has been reported elsewhere [29,30], which was confirmed through our experiments (Figure 7). Our results indicated mainly acute effects on HRV and ECG morphology features with an approximately $1 \mathrm{~h}$ recovery time. The effect was more pronounced in time-domain and nonlinear HRV variable (HR, RMSSD and SD1/SD2; Figure 6-8). HR recovery to the baseline level was slower than other variables and took place after approximately $1 \mathrm{~h}$. As shown in Figure 3C, human interaction can disrupt the autoregulation of cardiac function toward sympathetic modulation; reflected as early and sustained tachycardia here. This could mask elevated vagal tone that we might expect to observe on HRV by VNS. For instance, the HF band in frequency analysis of $\mathrm{HR}$ variations mainly reflects parasympathetic activity corresponding to the respiratory cycle [24]. LF/ HF trend in Figure 6C showed that VNS acutely disrupts sympathovagal balance (also mirrored in SD1/SD2) toward parasympathetic dominance for at least $30 \mathrm{~min}$. While it was not found to be significant, excluding the confounding human interaction factor might pronounce this effect. The SD1/SD2 is another measure for autonomic balance, particularly for short-term/long-term variation in the presence of sympathetic activation [24]. As shown in Figure 8A, short-term variability (related to HF component of HR) becomes predominant and significantly disrupts the balance for at least $30 \mathrm{~min}$ after VNS. Long-term effects of VNS were also shown here with some observable trends. However, the physiological interpretation remains to be seen with future studies using a larger sample size and comparing with a sham group.

Selected long-term VNS experiments using rat models were found from the literature and summarized in Table 1. Duration of VNS experiments for these studies ranged from 4 days to 12 weeks. Although the majority of reported studies applied VNS on awake-behaving animals, chronic but intermittent physiological monitoring 
was only reported in a few studies with the maximum experimental duration of 10 weeks [29,31-34]. However, the electrode integrity assessment was only performed in two studies using ad hoc observational assessment of physiological target engagement [29] or infrequent impedance measurement [33]. Compared with listed studies in Table 1, our suggested model provides round-the-clock physiological monitoring (here ECG) for long-term VNS experiments, which is critical for investigating closed-loop therapy [35] or effects on sleep and circadian cycles [36]. More importantly, weekly electrode impedance measurements were performed to confirm the durability of the implanted electrodes. The impedance trend in Figure 4 indicates that biotic and abiotic factors during the acute phase potentially influenced change in impedance of the electrode, which did not change substantially at later time points. However, it seems critical to continuously measure impedance throughout the course of the experiment to ensure electrode integrity for long-term use. For example, in one rat at week 16, we measured an abrupt increase in impedance $(>300 \mathrm{k} \Omega$ ) indicating electrode failure; whereas it did not change before the failure point. The use of an external stimulator may not be ideal for VNS delivery in awake-behaving animals, due to the amount of interaction and animal handling it requires. Perhaps with the use of an implantable or wearable neurostimulators, the chances of lead wire breakage can be reduced. At the end of the study, rats were euthanized, and cuff electrodes were harvested to ensure integrity. Although reported VNS rate is variable and ranged from few seconds [12] to few hours [37] per day, the proposed model can be used to implement short (but as frequent as desired) VNS sessions using awake rats.

Some limitations of the present study should be taken into consideration. We assessed this model only for short and intermittent VNS, but longer experimentation needs to be done for further validation. The magnetic connector system was susceptible to disconnection if animals moved rapidly. A stronger magnet or increasing the number of magnets may mitigate this concern. Here, we represented data for biweekly VNS, but daily or more frequent VNS needs to be done for elucidating the effects on cardiovascular system. As we observed from the HR trend shown in Figure 3C., the confounding effect of human interaction cannot be completely avoided using this system. Although we designed the connector mount in a way to minimize any interaction with the animal and handling, the presence of experimenter in the room can simply influence HR, disrupt the autoregulation mechanism of cardiac rhythms and consequently affect other cardiovascular variables. Complete exclusion of human interaction entails using wireless implantable stimulators, which is one of the scopes of our future studies. In this study, we used a limited number of animals. For that reason, we did not claim any physiological effects influenced by VNS. The goal here was to introduce our model and methodology that can be easily implemented in experiments with larger sample size and more efficient design (e.g., including sham group) for any physiological claims and statistical interpretation. Physical size, power requirement and cost are main limitations of telemetry implants for physiological monitoring in rodents. A battery is usually implanted along with the sensor to provide energy for wireless signal transmission which can increase the size of the device and limit the continuous signal recording in a chronic setup [41]. As indicated before, we lost ECG signals from two rats at the middle of the experiment. After troubleshooting with the manufacturer, we learned that there was a problem with the battery from that implant batch. However, we continued VNS on those animals and were able to verify the VNS target engagement based on behavioral response assessment (e.g., coughing).

\section{Conclusion}

An animal model for VNS was proposed and feasibility in a long-term experimental setup was evaluated. Results demonstrated the durability of physiological signals and implanted electrodes for chronic VNS experiments. Cardiovascular changes by VNS were also explored using ECG morphology and HRV analyses. Preliminary data for cardiovascular analysis revealed some transient effects during VNS. However, the confounding factor of human interaction on ECG features should be taken into the account before drawing any physiological conclusion on cardiovascular effects.

\section{Author contributions}

Conceptualization: F Yaghouby and S Vasudevan; device design and surgery: S Vasudevan; data collection: F Yaghouby and B Shafer; data analysis and manuscript preparation: F Yaghouby and S Vasudevan; funding acquisition: S Vasudevan.

\section{Acknowledgments}

The authors thank Sepideh Asgari for her assistance with experiments and data collection. 


\section{Disclaimer}

The mention of commercial products, their sources, or their use in connection with material reported herein is not to be construed as either an actual or implied endorsement of such products by the Department of Health and Human Services.

Financial \& competing interests disclosure

This research project was supported by the Defense Advanced Research Project Agency (DARPA), Biotechnology Technology Office (BTO), Targeted Neuroplasticity Training (TNT) program through an Interagency Agreement with the US FDA. An appointment was made to the Research Participation Program at the Center for Devices and Radiological Health (FDA) administered by the Oak Ridge Institute for Science and Education through an interagency agreement between the Department of Energy and FDA. The authors have no other relevant affiliations or financial involvement with any organization or entity with a financial interest in or financial conflict with the subject matter or materials discussed in the manuscript apart from those disclosed.

No writing assistance was utilized in the production of this manuscript.

\section{Ethical conduct of research}

The authors state that this study was approved by the Institutional Animal Care and Use Committee (IACUC) at the Food and Drug Administration, White Oak campus. They state that they have obtained appropriate institutional review board approval or have followed the principles outlined in the Declaration of Helsinki for all human or animal experimental investigations.

\section{Summary points}

- A rodent model to facilitate long-term VNS experiments for the assessment of safety and efficacy was developed.

- Feasibility of the model was validated by investigating long-term VNS effects on the cardiovascular system.

- Results shown easy-to-interface VNS system, electrode robustness and discernible ECG signals using unanesthetized rats in a chronic experimental setup.

\section{References}

Papers of special note have been highlighted as: $\bullet$ of interest; $\bullet \bullet$ of considerable interest

1. Yuan H, Silberstein SD. Vagus nerve and vagus nerve stimulation, a comprehensive review: part II. Headache 56(2), 259-266 (2016).

-• A thorough review for different aspects of vagus nerve stimulation (VNS) includying but not limited to anatomy, physiology, clinical and preclinical applications, future, etc.

2. Groves DA, Brown VJ. Vagal nerve stimulation: a review of its applications and potential mechanisms that mediate its clinical effects. Neurosci. Biobehav. Rev. 29(3), 493-500 (2005).

3. Lendvai IS, Maier A, Scheele D, Hurlemann R, Kinfe TM. Spotlight on cervical vagus nerve stimulation for the treatment of primary headache disorders: a review. J. Pain Res. 11, 1613-1625 (2018).

4. Koopman FA, Van Maanen MA, Vervoordeldonk MJ, Tak PP. Balancing the autonomic nervous system to reduce inflammation in rheumatoid arthritis. J. Intern. Med. 282(1), 64-75 (2017).

-• Importance of rodent sudies for translational use of VNS in clinical setup.

5. Vanneste S, Martin J, RennakerRL, 2nd, Kilgard MP. Pairing sound with vagus nerve stimulation modulates cortical synchrony and phase coherence in tinnitus: an exploratory retrospective study. Sci. Rep. 7(1), 17345 (2017).

6. Bonaz B, Sinniger V, Hoffmann D et al. Chronic vagus nerve stimulation in Crohn's disease: a 6-month follow-up pilot study. Neurogastroenterol. Motil. 28(6), 948-953 (2016).

7. Dawson J, Pierce D, Dixit A et al. Safety, feasibility, and efficacy of vagus nerve stimulation paired with upper-limb rehabilitation after ischemic stroke. Stroke 47(1), 143-150 (2016).

8. Cimpianu CL, Strube W, Falkai P, Palm U, Hasan A. Vagus nerve stimulation in psychiatry: a systematic review of the available evidence. J. Neural Transm. (Vienna) 124(1), 145-158 (2017).

9. Hays SA, Rennaker RL, Kilgard MP. Targeting plasticity with vagus nerve stimulation to treat neurological disease. Prog. Brain Res. 207, 275-299 (2013).

10. Loerwald KW, Buell EP, Borland MS, Rennaker RL, 2nd, Hays SA, Kilgard MP. Varying stimulation parameters to improve cortical plasticity generated by VNS-tone pairing. Neuroscience 388, 239-247 (2018).

11. Engineer CT, Engineer ND, Riley JR, Seale JD, Kilgard MP. Pairing speech sounds with vagus nerve stimulation drives stimulus-specific cortical plasticity. Brain Stimul. 8(3), 637-644 (2015).

- Proposes safe VNS parameters for use in rodent models. 
12. Meyers EC, Solorzano BR, James J et al. Vagus nerve stimulation enhances stable plasticity and generalization of stroke recovery. Stroke 49(3), 710-717 (2018).

13. Noble LJ, Gonzalez IJ, Meruva VB et al. Effects of vagus nerve stimulation on extinction of conditioned fear and post-traumatic stress disorder symptoms in rats. Transl. Psychiatry 7(8), e1217 (2017).

14. Engineer CT, Hays SA, Kilgard MP. Vagus nerve stimulation as a potential adjuvant to behavioral therapy for autism and other neurodevelopmental disorders. J. Neurodev. Disord. 9, 20 (2017).

15. Aalbers M, Vles J, Klinkenberg S, Hoogland G, Majoie M, Rijkers K. Animal models for vagus nerve stimulation in epilepsy. Exp. Neurol. 230(2), 167-175 (2011).

16. Levine YA, Koopman FA, Faltys $\mathrm{M}$ et al. Neurostimulation of the cholinergic anti-inflammatory pathway ameliorates disease in rat collagen-induced arthritis. PLoS ONE 9(8), e104530 (2014).

17. Andersson U, Tracey KJ. Neural reflexes in inflammation and immunity. J. Exp. Med. 209(6), 1057-1068 (2012).

18. Ben-Menachem E. Modern management of epilepsy: vagus nerve stimulation. Baillieres Clin. Neurol. 5(4), 841-848 (1996).

19. Sun P, Wang J, Zhao $S$ et al. Improved outcomes of cardiopulmonary resuscitation in rats treated with vagus nerve stimulation and its potential mechanism. Shock 49(6), 698-703 (2018).

20. Loeb GE, Peck RA. Cuff electrodes for chronic stimulation and recording of peripheral nerve activity. J. Neurosci. Methods 64(1), 95-103 (1996).

21. Li P, Liu H, Sun $\mathrm{P}$ et al. Chronic vagus nerve stimulation attenuates vascular endothelial impairments and reduces the inflammatory profile via inhibition of the NF-kappaB signaling pathway in ovariectomized rats. Exp. Gerontol. 74, 43-55 (2016).

22. Straka MM, Shafer B, Vasudevan S, Welle C, Rieth L. Characterizing longitudinal changes in the impedance spectra of in-vivo peripheral nerve electrodes. Micromachines 9(11), 587-607 (2018).

23. Vasudevan S, Patel K, Welle C. Rodent model for assessing the long term safety and performance of peripheral nerve recording electrodes. J. Neural Eng. 14(1), 016008 (2017).

- Addresses the significance of long-term animal experiments for assessment of safety and performance for neurological implants.

24. Shaffer F, Ginsberg JP. An overview of heart rate variability metrics and norms. Front. Public Health 5, 258 (2017).

25. Al Dahhan NZ, De Felice FG, Munoz DP. Potentials and pitfalls of cross-translational models of cognitive impairment. Front. Behav. Neurosci. 13, 48 (2019).

26. Musselman ED, Pelot NA, Grill WM. Empirically based guidelines for selecting vagus nerve stimulation parameters in epilepsy and heart failure. Cold Spring Harb. Perspect. Med. 9(7), pii: a034264 (2018).

27. Hays SA. Enhancing rehabilitative therapies with vagus nerve stimulation. Neurotherapeutics 13(2), 382-394 (2016).

28. Caravaca AS, Tsaava T, Goldman L et al. A novel flexible cuff-like microelectrode for dual purpose, acute and chronic electrical interfacing with the mouse cervical vagus nerve. J. Neural Eng. 14(6), 066005 (2017)

29. Chapleau MW, Rotella DL, Reho JJ, Rahmouni K, Stauss HM. Chronic vagal nerve stimulation prevents high-salt diet-induced endothelial dysfunction and aortic stiffening in stroke-prone spontaneously hypertensive rats. Am. J. Physiol. Heart Circ. Physiol. 311(1), H276-285 (2016).

30. Lee SW, Kulkarni K, Annoni EM, Libbus I, Kenknight BH, Tolkacheva EG. Stochastic vagus nerve stimulation affects acute heart rate dynamics in rats. PLoS ONE 13(3), e0194910 (2018).

31. Li M, Zheng C, Sato T, Kawada T, Sugimachi M, Sunagawa K. Vagal nerve stimulation markedly improves long-term survival after chronic heart failure in rats. Circulation 109(1), 120-124 (2004).

32. Annoni EM, Van Helden D, Guo Y et al. Chronic low-level vagus nerve stimulation improves long-term survival in salt-sensitive hypertensive rats. Front. Physiol. 10, 25 (2019).

33. Dedeurwaerdere S, Gilby K, Vonck K, Delbeke J, Boon P, Mcintyre D. Vagus nerve stimulation does not affect spatial memory in fast rats, but has both anti-convulsive and pro-convulsive effects on amygdala-kindled seizures. Neuroscience 140(4), 1443-1451 (2006).

34. Yoshida K, Saku K, Kamada K et al. Electrical vagal nerve stimulation ameliorates pulmonary vascular remodeling and improves survival in rats with severe pulmonary arterial hypertension. JACC Basic Transl. Sci. 3(5), 657-671 (2018).

-• Tries to study how VNS can affect hemodynamic variables such as blood pressure in rodents and explore theraputic effects of VNS for pulmonary arterial hypertension.

35. Ganzer PD, Sharma G. Opportunities and challenges for developing closed-loop bioelectronic medicines. Neural Regeneration Res. 14(1), 46-50 (2019).

36. Daley JT, Dewolfe JL. Sleep, circadian rhythms, and epilepsy. Curr. Treat. Options Neurol. 20(11), 47 (2018).

37. Sun $\mathrm{P}$, Zhou K, Wang $\mathrm{S}$ et al. Involvement of MAPK/NF-kappaB signaling in the activation of the cholinergic anti-inflammatory pathway in experimental colitis by chronic vagus nerve stimulation. PLoS ONE 8(8), e69424 (2013).

38. Pena DF, Childs JE, Willett S, Vital A, Mcintyre CK, Kroener S. Vagus nerve stimulation enhances extinction of conditioned fear and modulates plasticity in the pathway from the ventromedial prefrontal cortex to the amygdala. Front. Behav. Neurosci. 8, 327 (2014). 
39. Zhang $Y$, Chen A, Song $L$ et al. Low-level vagus nerve stimulation reverses cardiac dysfunction and subcellular calcium handling in rats with post-myocardial infarction heart failure. Int. Heart J. 57(3), 350-355 (2016).

- Investigates cardiac effects of VNS in rat models of heart failure.

40. Farrand AQ, Helke KL, Gregory RA, Gooz M, Hinson VK, Boger HA. Vagus nerve stimulation improves locomotion and neuronal populations in a model of Parkinson's disease. Brain Stimul. 10(6), 1045-1054 (2017).

41. Niemeyer JE. Telemetry for small animal physiology. Lab. Animal 45, 255 (2016).

- Discusses benefits and chalanges related to use of wireless physiologial monitoring devices in small animal models. 